\title{
Lysozyme's lectin-like characteristics facilitates its immune defense function
}

\author{
Ruiyan Zhang ${ }^{1,2}$, Lisha $\mathrm{Wu}^{3}$, Thomas Eckert ${ }^{4,5}$, Monika Burg-Roderfeld ${ }^{5}$, Miguel A. Rojas-Macias ${ }^{5}$, \\ Thomas Lütteke ${ }^{5}$, Vadim B. Krylov ${ }^{6}$, Dmitry A. Argunov ${ }^{6}$, Aritreyee Datta ${ }^{7}$, Philipp Markart ${ }^{8,9}$, \\ Andreas Guenther ${ }^{10}$, Bengt Norden ${ }^{3}$, Roland Schauer ${ }^{9}$, Anirban Bhunia ${ }^{7}$, Mushira Abdelaziz Enani ${ }^{11}$, \\ Martin Billeter ${ }^{12 *}$, Axel J. Scheidig ${ }^{2 *}$, Nikolay E. Nifantiev ${ }^{6 *}$ and Hans-Christian Siebert ${ }^{1 *}$ \\ ${ }^{1}$ RI-B-NT Research Institute of Bioinformatics and Nanotechnology, Franziusallee 177, 24148 Kiel, Germany \\ ${ }^{2}$ Department of Structural Biology, Institute of Zoology, Christian-Albrechts-University, Am Botanischen Garten 1-9, 24118 Kiel, Germany \\ ${ }^{3}$ Department of Chemical and Biological Engineering, Chalmers University of Technology, 41296 Gothenburg, Sweden \\ ${ }^{4}$ Clinic of Obstetrics, Gynecology and Andrology for Small and Large Animals, Justus-Liebig-University, Justus-Liebig-University Giessen, Frankfurter Str. \\ 106, 35392 Giessen, Germany \\ ${ }^{5}$ Institute for Veterinary Physiology and Biochemistry, Justus-Liebig-University, Frankfurter Str.100, 35392 Giessen, Germany \\ ${ }^{6}$ Laboratory of Glycoconjugate Chemistry, N.D. Zelinsky Institute of Organic Chemistry, Russian Academy of Sciences, Leninsky prospect 47,119991 \\ Moscow, Russian Federation \\ ${ }^{7}$ Department of Biophysics, Bose Institute, P-1/12 CIT Scheme VII (M), Kolkata 700054, India \\ ${ }^{8}$ Pneumology, Heart-Thorax-Center Fulda, Pacelliallee 4-36043 Fulda, Germany \\ ${ }^{9}$ Institute of Biochemistry, Christian-Albrechts-University, Olshausenstrasse 40, 24098 Kiel, Germany \\ ${ }^{10}$ Medical Clinic II, Justus-Liebig-University, Klinikstraße 33, 35392 Giessen, Germany; Member of the German Center for Lung Research (DZL) \\ ${ }^{11}$ Infectious Diseases Division, Department of Medicine, King Fahad Medical City, PO Box 59046, Riyadh 11525, Kingdom of Saudi Arabia \\ ${ }^{12}$ Department of Chemistry and Molecular Biology, University of Gothenburg, 40530 Gothenburg, Sweden
}

Quarterly Reviews of Biophysics (2017), 50, e9, page 1 of 12 doi:10.1017/S0033583517000075

Abstract. Interactions between human lysozyme (HL) and the lipopolysaccharide (LPS) of Klebsiella pneumoniae O1, a causative agent of lung infection, were identified by surface plasmon resonance. To characterize the molecular mechanism of this interaction, HL binding to synthetic disaccharides and tetrasaccharides representing one and two repeating units, respectively, of the O-chain of this LPS were studied. $\mathrm{pH}$-dependent structural rearrangements of $\mathrm{HL}$ after interaction with the disaccharide were observed through nuclear magnetic resonance. The crystal structure of the HL-tetrasaccharide complex revealed carbohydrate chain packing into the A, B, C, and D binding sites of HL, which primarily occurred through residue-specific, direct or water-mediated hydrogen bonds and hydrophobic contacts. Overall, these results support a crucial role of the Glu35/Asp53/Trp63/Asp102 residues in HL binding to the tetrasaccharide. These observations suggest an unknown glycan-guided mechanism that underlies recognition of the bacterial cell wall by lysozyme and may complement the HL immune defense function.

\footnotetext{
* Authors for correspondence: M. Billeter, Department of Chemistry and Molecular Biology, University of Gothenburg, Box 462, 40530 Gothenburg, Sweden. Tel: +46 31 7863925; Fax: +46 31 7862599; Email: martin.billeter@ chem.gu.se

A. J. Scheidig, Department of Structural Biology, Institute of Zoology, Christian-Albrechts-University, Am Botanischen Garten 1-9, 24118 Kiel, Germany. Tel: +49 431 8804286; Fax: +49 431 8804929; Email: axel.scheidig@ strubio.uni-kiel.de.

N. E. Nifantiev, N.D. Zelinsky Institute of Organic Chemistry, Russian Academy of Sciences, Leninsky prospect 47, 119991 Moscow, Russian Federation. Tel: +7 499 1358784; Fax: +7 499 1358784; Email: nen@ioc.ac.ru H-C. Siebert, RI-B-NT Research Institute of Bioinformatics and Nanotechnology, Franziusallee 177, 24148 Kiel, Germany. Tel.: +49 431 66878443; Fax: +49 4315606 295; Email: hcsiebert@aol.com
}

\section{Introduction}

Lower respiratory tract infections are among the top 10 causes of death worldwide and are of particular relevance in chronic lung diseases. Lysozyme is one of the most abundant antimicrobial proteins in the airways and alveoli. The concentration of this enzyme in the surface liquid of the human airway is estimated to be $20-100 \mu \mathrm{g} \mathrm{ml}^{-1}$, which is sufficient to kill important pulmonary pathogens such as Gram-positive Staphylococcus aureus and Gram-negative Pseudomonas aeruginosa (Travis et al. 1999). Klebsiella pneumoniae, which is a frequent cause of nosocomial 
infection and may be responsible for up to $20 \%$ of the respiratory infections in neonatal intensive care units (Gupta, 2002), is also specifically attacked by lysozyme (Markart et al. 2004).

Human lysozyme (HL, also known as muramidase, $N$-acetyl muramide glycanohydrolase, or EC 3.2.1.17; Fig. 1a) is a 130 -amino acid cationic protein that cleaves the glycosidic bonds of $\mathrm{N}$-acetyl-muramic acid (Fig. $1 c$ ), thereby damaging the bacterial cell wall (Fig. $1 b$ ) and ultimately killing bacteria by lysis in cooperation with defensins. An inter-domain cleft in HL contains six binding pockets (labeled A-F in Fig. 1a), and a strictly conserved catalytic residue Glu35 is located between the D and E sites (Chipman \& Sharon, 1969). Co-crystallization of $\mathrm{HL}$ with $\mathrm{N}$-acetyl-chitohexaose $($ GlcNAc) 6 (Fig. 1c) has revealed a crystal structure with non-covalently linked (GlcNAc) $)_{4}$ and $(\mathrm{GlcNAc})_{2}$ in subsites A-D and E-F, respectively (Song et al. 1994).

In addition to the well-known muramidase activity of HL, increasing evidence suggests the existence of non-enzymatic and/or nonlytic modes of action against Gram-negative and Gram-positive bacteria (Lee-Huang et al. 2005; Masschalck \& Michiels, 2003). Furthermore, lysozyme has antitumor (Osserman et al. 1973) and antiviral activities (Lee-Huang et al. 2005), and it enhances the immune system (Siwicki et al. 1998). The mechanisms of these activities remain unclear, and the dominant questions involve how HL recognizes pathogenic microbes.

Bacterial LPSs represent the group of important virulence factors, which are recognized by antibodies and pattern recognition receptors in the initial steps of innate immune response to Gram-negative bacteria. These processes and their physico-chemical characteristics were previously studied using surface plasmon resonance (SPR)-method (Shin et al. 2007; Young et al. 1999). The ability of lysozyme to interact with lipopolysaccharides (LPSs) was demonstrated in the late 1980s (Ohno \& Morrison, 1989a, b). However, the details of this interaction (i.e. how binding specificity is established between specific parts of the lysozyme protein and the LPS carbohydrate units) have not been examined. The attraction between lysozyme and LPS has been largely attributed to non-specific hydrophobic interactions of lysozyme with lipid A, which is the innermost hydrophobic component of LPS and is primarily responsible for its toxicity. To examine whether lysozyme specifically interacts with bacterial LPS and particularly with the O-chains that form the outer layer of the bacterial cell wall, we performed SPR-based experiments as previously described (Tsvetkov et al. 2012). By SPR experiments we detected the interaction between HL and LPS from K. pneumoniae O1, which is associated with the development of severe hospital-acquired infections and is clinically relevant to infections beyond those of the airways (Enani, 2015; Enani \& El-Khizzi, 2012). SPR permitted to measure corresponding dissociation constant $\left(K_{\mathrm{d}}\right)$ of $0.41 \mathrm{mM}$ and association and dissociation rate constants $\left(k_{\mathrm{a}}\right.$ and $\left.k_{\mathrm{d}}\right)$ of $216 \mathrm{M}^{-1} \mathrm{~s}^{-1}$ and $0.0886 \mathrm{~s}^{-1}$, respectively (online Fig. S1 in Supplementary Information). To assess whether the O-chains of these LPSs were involved in the observed interactions, we combined nuclear magnetic resonance (NMR), molecular modeling, data mining and X-ray crystallography techniques to investigate HL binding to synthetic disaccharide 1 (Krylov et al. 2014) and tetrasaccharide 2 (Fig. 1d), which represented one and two repeating units of the O-chain of K. pneumoniae O1, respectively, at the sub-molecular level.

\section{Materials and methods}

\subsection{Lysozyme}

Recombinant HL was provided by T. E. Weaver (Cincinnati Children's Hospital Medical Center, Cincinnati, USA). The HL was purified as previously described for other lysozymes (Akinbi et al. 2000; Markart et al. 2004).

\subsection{Oligosaccharides}

The synthesis of disaccharide $\mathbf{1}$ was performed using the recently discovered pyranoside-into-furanoside rearrangement (Krylov et al. 2014, 2016). The synthesis of tetrasaccharide 2 (Verkhnyatskaya et al. 2017) was based on similar approaches and is described in the online supplementary information (Scheme S1).

\subsection{SPR analysis}

SPR was performed on a protein interaction array system (ProteOn XPR36, Bio-Rad, Munich, Germany). Vertical channels of a GLM sensor chip were activated with undiluted EDAC-sNHS 1:1 mixtures according to the amine coupling kit (Biorad). HL was immobilized from $100 \mu \mathrm{g}$ $\mathrm{ml}^{-1}$ solutions in $10 \mathrm{mM}$ acetate buffer $\mathrm{pH} 4.5$ on the activated sides of channel. Remaining active sides of the channels were blocked by treatment with $1 \mathrm{M}$ ethanolamine. All steps were performed in phosphate buffered saline $\mathrm{pH} 7 \cdot 4$ containing $0 \cdot 005 \%$ Tween 20 (PBS-T) running buffer using a flow rate of $30 \mu \mathrm{l} \mathrm{min}{ }^{-1}$ and $150 \mu \mathrm{l}$ total reaction volume, respectively. A regeneration of the sensor surface was performed before each testing by injecting $0.85 \%$ phosphoric acid solution at $100 \mu \mathrm{min}^{-1}$ for $18 \mathrm{~s}$.

LPS of Klebsiella was diluted in running buffer and different concentrations $(10,20,40,80 \mu \mathrm{M})$ were injected for $60 \mathrm{~s}$ association time corresponding to a total reaction volume of $100 \mu \mathrm{l}$ at $100 \mu \mathrm{l} \mathrm{min}{ }^{-1}$ followed by $600 \mathrm{~s}$ dissociation step using an equal flow rate via channels A1-A4 (horizontal channels). Interaction binding signal (RU) raw data of channels were referenced by subtraction of the unspecific binding signals on uncovered channel. Double reference was carried out by subsequent subtraction of unspecific binding signals measured in a reference channel with 
(a)

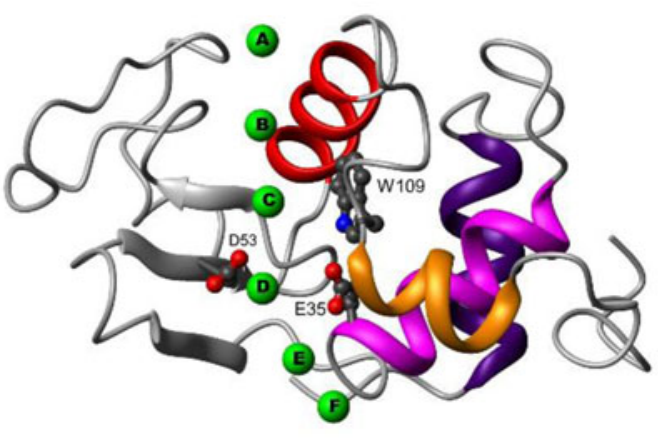

(b)

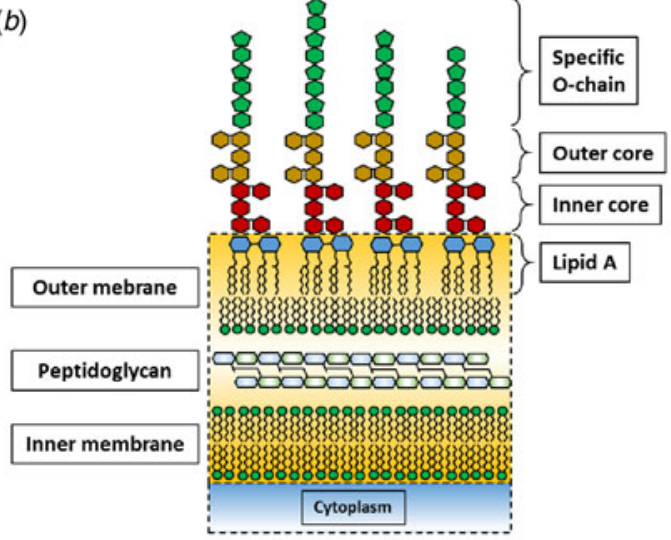

(c)

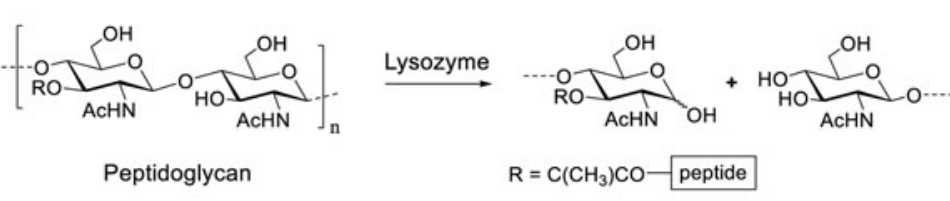

Peptidoglycan

$\mathrm{R}=\mathrm{C}\left(\mathrm{CH}_{3}\right) \mathrm{CO}-$ peptide (d)

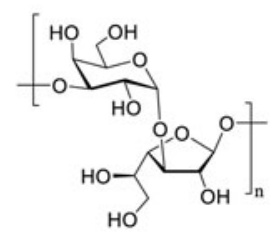

O-Chain repeating unit

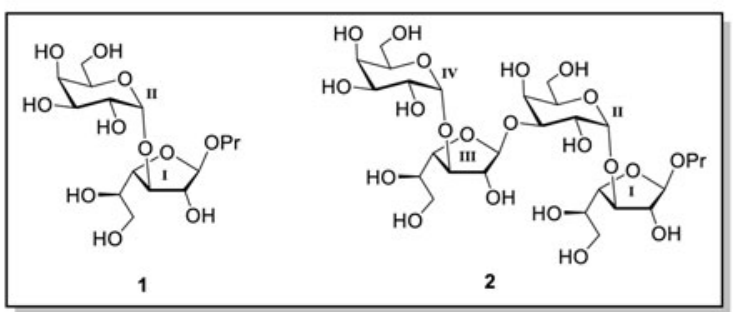

Fig. 1. Lysozyme and its carbohydrate ligands (a) Structure of HL (PDB code 1LZS) with selected residues (Glu35, Asp53, and Trp109). Binding sites A-F are indicated by letters in green circles. Helices are colored as follows: $a$, violet; $b$, magenta; $c$, red; $d$, orange. In the orientation shown, the $\alpha$-domain is to the right of the binding cleft and the $\beta$-domain is to the left. (b) Schematic drawing of the LPS molecule and its position in the outer layer of the outer membrane of Gram-negative bacteria; for clarity, membrane associated proteins and integral membrane proteins are not shown. $(c)$ Hydrolysis of the $(1 \rightarrow 4)$-glycosidic bond between $\mathrm{N}$-acetyl muramic acid and $\mathrm{N}$-acetyl glucosamine in the peptidoglycan. (d) Structures of the repeating unit of the O-chain of the K. pneumoniae O1 lipopolysaccharide and the structurally related synthetic disaccharide $\mathbf{1}$ and tetrasaccharide $\mathbf{2}$. Monosaccharide units are numbered with Roman numerals.

PBS-T. Data were analyzed with the computer software (ProteOn manager software, Bio-Rad). Kinetic constants were calculated using Langmuir model kinetic analysis of the ProteOn manager software (Bio-Rad).

\subsection{NMR sample preparation}

All samples were prepared by dissolving lyophilized HL in $0.3 \mathrm{ml} \mathrm{H}_{2} \mathrm{O}$ containing $20 \mathrm{mM}$ sodium phosphate buffer and $10 \% \mathrm{D}_{2} \mathrm{O}$. Final concentrations of all samples were $0.5 \mathrm{mM}$ protein as determined by measurement of the molar extinction coefficient, using E1\% $=25.5$ for HL. For mixtures of lysozyme with disaccharide 1, a $40 \mathrm{mM}$ stock solution was prepared. The following samples were prepared: pure HL as well as 1:1 mixtures of these proteins with disaccharidel at $\mathrm{pH} 3.8$ and 5.5. Additional samples for HL were prepared in a similar manner for the $\mathrm{pH}$ titrations described below.

\subsection{NMR measurements}

All NMR spectra were recorded on a Varian Unity INOVA $800 \mathrm{MHz}$ spectrometer at $35^{\circ} \mathrm{C}$ using $3 \mathrm{~mm}$ Shigemi tubes. The ${ }^{1} \mathrm{H}$ chemical shifts were referenced to DSS. For disaccharide-lysozyme interaction measurements, equivalent amounts of disaccharidel were added to the enzymes. Homonuclear 2D NOESY (mixing time $150 \mathrm{~ms}$ ) and TOCSY (mixing time $50 \mathrm{~ms}$ ) with spectral width 11204 $\mathrm{Hz}$ for both dimensions were acquired with 512 increments in the indirect dimension and 4096 data points in the direct dimension, using Watergate solvent suppression and a pulse sequence repetition delay of $1.5 \mathrm{~s}$. All data were processed with the NMRPipe software package (Delaglio et al. 1995) by zero-filling to 1024 points in the indirect dimension and ending with either a Gaussian or a shifted sine-bell function. After zero-filling the digital resolution was $0.0015 \mathrm{ppm}$ for the direct dimension and $0.014 \mathrm{ppm}$ for 
the indirect dimension. For pKa determinations of HL, 10 NOESY spectra were recorded in the $\mathrm{pH}$ range $3 \cdot 8-8 \cdot 1$ $(3 \cdot 8,4 \cdot 2,4 \cdot 6,5 \cdot 0,5 \cdot 5,6 \cdot 2,6 \cdot 8,7 \cdot 4,7 \cdot 7$ and $8 \cdot 1)$. A separate sample of HL was prepared for recording of $221 \mathrm{D}$ spectra covering the $\mathrm{pH}$ range of $3 \cdot 17-8.13$ in steps of $\sim 0.2$ units. All 1D datasets were defined by 4096 complex points and consisted of 256 transients. The digital resolution of the $1 \mathrm{D}$ spectra was $0.0024 \mathrm{ppm}$ after zero-filling. Xeasy (Bartels et al. 1995), Mnova (Claridge, 2009), and CCPNmr (Vranken et al. 2005) were used for analysis and resonance assignment. Line widths are defined as half-width at half-height of a peak; for most peaks the line width was estimated to be $0.01 \mathrm{ppm}$.

All $\mathrm{pH}$ adjustments were made by addition of small aliquots of either $\mathrm{H}_{3} \mathrm{PO}_{4}$ or $\mathrm{NaOH}$. The $\mathrm{pH}$ meter was calibrated with standard solutions (from Sigma) at $\mathrm{pH} 4$ and 7 . The temperature dependence of the $\mathrm{pH}$ reading for $\mathrm{HL}$ was checked by recalibrating the $\mathrm{pH}$ meter at $35^{\circ} \mathrm{C}$ : the difference between an incubated lysozyme sample at $35^{\circ} \mathrm{C}$ and at room temperature was less than $0.1 \mathrm{pH}$ unit. The $\mathrm{pH}$ for each sample was measured before and after each experiment to warrant constant conditions.

\subsection{Signal assignments}

${ }^{1} \mathrm{H}$ resonance assignments at $\mathrm{pH} 3.8$ and $\mathrm{pH} 5 \cdot 5$, both at $35^{\circ} \mathrm{C}$, were obtained for HL by transferring chemical shifts from the Biological Magnetic Resonance Bank (BMRB, http://www.bmrb.wisc.edu/) (Ulrich et al. 2008) entries 5130 and 1093, respectively, to the NOESY and TOCSY spectra. Based on chemical shift changes, conclusions about interactions between lysozymes and disaccharide $\mathbf{1}$ could be made in several cases as described in the main text. However, no intermolecular NOEs were detectable, suggesting dynamic binding modes only.

\subsection{Molecular modeling and data mining}

Docking studies were performed with the AutoDock $4 \cdot 2$ software, which uses the Lamarckian Genetic Algorithm (LGA) implemented therein. For the docking of the LPS disaccharide fragment 1 with human or chicken lysozyme, the required file for the ligand was created by combining the Gaussian and AutoDock 4.2 software packages. The grid size was set to 126,126 , and $126 \AA$ along the $X$-, $Y$-, and $Z$-axis; in order to recognize the LPS glycan binding site of HL, the blind docking simulation was adopted. The docking parameters used were the following: LGA population size $=150$; maximum number of energy evaluations $=250000$. The lowest binding energy conformer was taken from 10 different conformations for each docking simulation and the resultant minimum energy conformation was applied for further analysis. The MOLMOL (Koradi et al. 1996) and PyMOL (DeLano, 2002) software packages were applied for visualization and analysis of the docked complex. Data mining of protein-carbohydrate interactions in the Protein Data Bank (PDB) was performed with GlyVicinity at a redundancy level of 70\% (www. glycosciences.de/tools/glyvicinity/). Only non-covalently bound ligands in structures with a resolution of at least $3 \AA$ have been considered. The analysed dataset comprised 498 amino acids that have been found within a $4 \AA$ radius of 73 alpha-galactose residues in 73 different PDB entries.

\section{$2.8 X$ Xray}

For crystallization, the hanging-drop vapor-diffusion method was performed in 24-well plates. Single crystals of the HL were obtained by mixture of $2 \mu \mathrm{l}$ of the reservoir solution $(0.8 \mathrm{M} \mathrm{NaCl}, 25 \mathrm{mM} \mathrm{NaOAc}, \mathrm{pH} 4 \cdot 4-5 \cdot 6)$ with $2 \mu \mathrm{l}$ of the protein solution $\left(50 \mathrm{mg} \mathrm{ml}^{-1}\right.$ in $100 \mathrm{mM} \mathrm{NaCl}, 10 \mathrm{mM}$ phosphate buffer, $\mathrm{pH}$ 6.0). These drops were equilibrated against $1 \mathrm{ml}$ of the reservoir solution at $291 \mathrm{~K}$. For co-crystallization, the protein solution was mixed with a 10-fold excess of tetrasaccharide 2 and incubated for 30 min at room temperature prior to setting up the crystallization drop. Data collection for X-ray diffraction was performed at $100 \mathrm{~K}$. The crystals were transferred into liquid nitrogen for flash-cooling without the prior addition of cryoprotectants. All data processing was performed using the XDS/XSCALE (Kabsch, 2010) program package. Molecular replacement was carried out using the MOLREP (Vagin \& Teplyakov, 1997) program with the HL structure (PDB id: 1REX) (Muraki et al. 1996) as the search model. Model building and refinement were performed with the Refmac5 program as implemented in the CCP4 suite (Murshudov et al. 2011; Winn et al. 2011) and PHENIX (Adams et al. 2010). The COOT (Emsley et al. 2010) graphics program was used to interpret the electron density maps and to rebuild the structure.

\section{Results}

\subsection{NMR observations of structural rearrangements of the binding site of $H \mathrm{~L}$}

The lysozyme enzymatic reaction requires an initial protonated form of the highly conserved Glu35, which exhibits a high pKa of 6.8 (Kuramitsu et al. 1974). Two pH-based NMR titrations of lysozyme without (see below and Fig. 2) and with (see below and Fig. 3) the weakly binding disaccharide 1 were used to provide atomistic insights into the relationship between the (de)protonation of Glu35 and its associated structural rearrangements in the surrounding helices, which were suspected to control the continuation of the catalysis reaction and hence influence specificity.

The $\mathrm{pH}$ dependence of the lysozyme binding site modulations was analyzed for free lysozyme and lysozyme in the presence of the weakly binding disaccharide 1 (Fig. 1d), and conclusions regarding the interactions between lysozyme and disaccharide $\mathbf{1}$ were made on the basis of chemical 


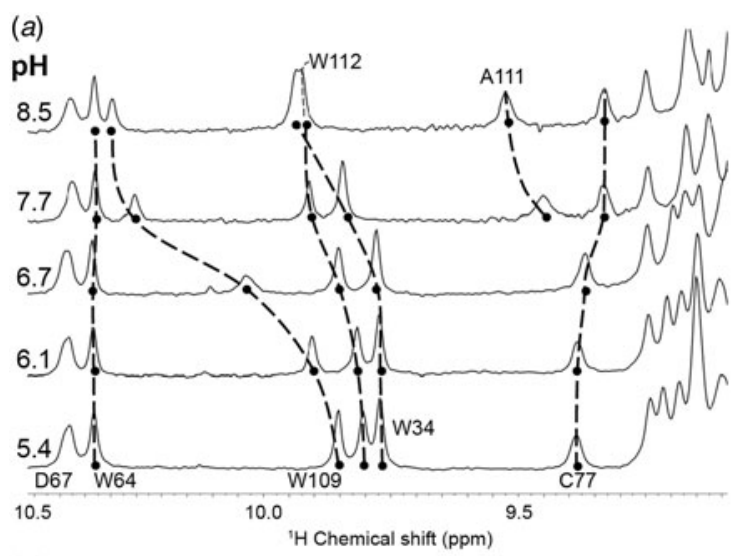

(b)

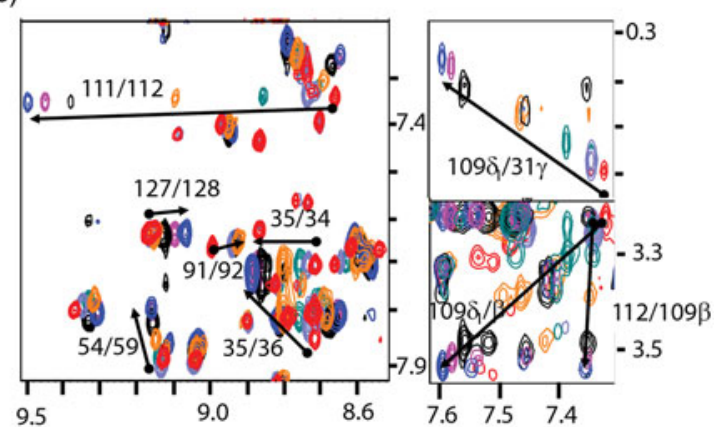

(c)

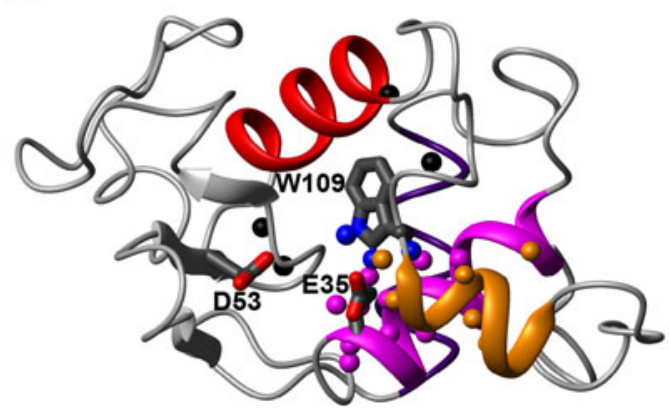

Fig. 2. NMR-based investigation of free human lysozyme (a) 1D spectra of pure HL at various $\mathrm{pH}$ values (indicated on the left border). For clarity, we show the residues, indole ring proton of Trp (Trp109, Trp112, and Trp34) and the HN of Cys77 and Ala111 (shown in top two traces), which show strong chemical shift perturbation. (b) Selected 2D NOESY regions for HL at various $\mathrm{pH}$ values. Spectra for the different $\mathrm{pH}$ values are colored as follows: $3 \cdot 8$, red; $5 \cdot 0$, light blue; $5 \cdot 5$, green; $6 \cdot 8$, orange; $7 \cdot 4$, black; $7 \cdot 7$, purple; 8.1 , dark blue. Chemical shifts that varied with $\mathrm{pH}$ are indicated by arrows on or beside the corresponding peaks with different colors. Peak contours are calibrated such that the intensities of the HN-HN cross-peaks for helix c are constant across all $\mathrm{pH}$ values. (c) Epitope mapping of $\mathrm{HL}$ from the $\mathrm{pH}$ titration, based on all resonances listed in the first part of online Table S1. Side chains are shown and labeled for Glu35, Asp53 and Trp109. Spheres are color-coded as follows: atoms on helix b are magenta, atoms on helix $\mathrm{d}$ are orange, and Trp109 side-chain atoms are blue $(\mathrm{H} \beta 2, \mathrm{H} \delta 1$ and $\mathrm{H} \varepsilon 1)$. Additionally, black spheres mark the HN positions from the following residues: 58 and 59 (near D53), 100 (at the end of the red helix c), and HN 108 (before Trp109). The structure is rotated by $30^{\circ}$ around a vertical axis with respect to Fig. $1 a$; the helix coloring is the same, and helix a is presented as a thin violet curve for clarity. shift changes. However, intermolecular NOEs between these two molecules were undetectable, thus suggesting the existence of only dynamic binding modes for which no stable structure could be determined. The results were therefore compared with the stable complex of lysozyme with tetrasaccharide 2 (Fig. 1d), which occupies four binding sites $(\mathrm{A}, \mathrm{B}, \mathrm{C}$, and D) (Fig. 1a).

\subsection{NMR-based investigation of free human lysozyme}

Titrations in the 3.8-8.5 $\mathrm{pH}$ range were observed for free HL by $1 \mathrm{D}$ and $2 \mathrm{D}$ NMR (online Table S1, Supplementary Information). Figure $2 a$ shows $1 \mathrm{D}$ spectra for different $\mathrm{pH}$ values, illustrating that the indole ring proton $(\mathrm{H} \epsilon 1)$ of residues Trp34, Trp109, Trp112, and the amide protons of Cys77 and Ala111 are strongly affected by $\mathrm{pH}$. The most pronounced change was observed for Trp109, with a shift to lower field by $0.43 \mathrm{ppm}$, whereas Trp64 and the overlapping Trp28 (at $9.13 \mathrm{ppm}$, not shown) did not titrate in the $3.8-8.5 \mathrm{pH}$ range. 2D NOESY spectra complemented the 1D titrations and provided a comprehensive picture of the chemical and structural changes in the enzyme (Fig. 2b). Again, large changes were observed for the $\mathrm{HN}$ of Ala111 (around $\mathrm{pH}$ 6.8) and for various side-chain atoms of Trp109. Additional chemical shift perturbations were also observed in large portions of helices $b$ and $d$ (Fig. 1a). The observed effects outside of these two helices included Gln58, Ile59, Val100 and Ala108 (online Table S1, Supplementary Information).

The strongest shift changes in the spatial neighborhood of the catalytic residue Glu35 were revealed by mapping of these shift changes onto the 3D structure of HL; they affect most of the $\mathrm{b}$ and $\mathrm{d}$ helices, and the loop between the second and third $\beta$-strands from the $\beta$-domain (Fig. $2 c$ ). Changes involving the HNs of residues Gln58, Ile59, Val100 and Ala108, were located in the plane of the Trp109 ring, thus indicating rotation of this ring. The common $\mathrm{pKa}$ value (within the measurement error) of all these resonances, about $\mathrm{pH} 6.8$, coincided with the reported (and unusually high) $\mathrm{pKa}$ value of the catalytic Glu35 protonation site (Kuramitsu et al. 1974); this strongly indicated that all of these events are coupled (online Table S1 and Fig. S2 and S3 Supplementary Information). The common pKa value (within the measurement error) of all these resonances, about $\mathrm{pH} 6 \cdot 8$, coincided with the reported (and unusually high) $\mathrm{pKa}$ value of the catalytic Glu35 protonation site (Kuramitsu et al. 1974), and furthermore identical pKa strongly indicated that all of these events are coupled (online Table S1, Supplementary Information). The large shift variation of $\sim 0.84 \mathrm{ppm}$ for the $\mathrm{HN}$ of Ala111 is probably the result of an adjacent charge change, and the obvious cause of this shift variation is the Glu35 side chain, whose carboxyl group is nearby at $3.4 \AA$ (Harata et al. 1998). The remarkable behavior of the $\mathrm{HN}$ of Ala111 was an important observation that demonstrated a direct coupling between helices $b$ and 
(a)

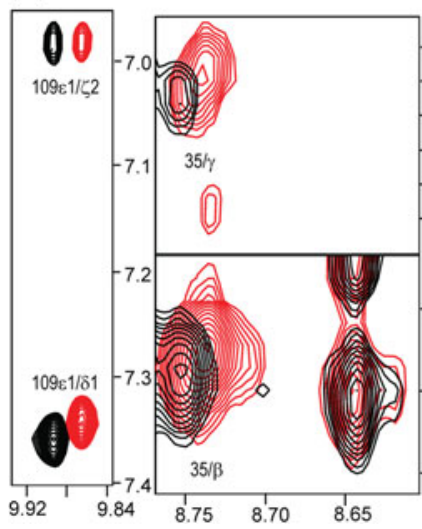

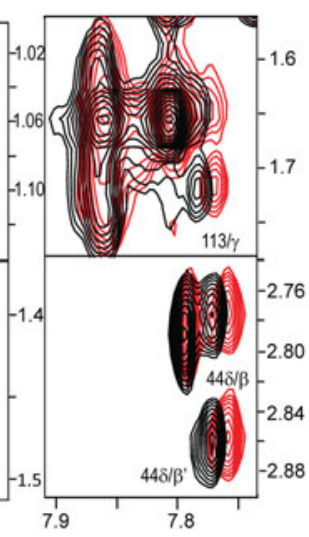

(b)

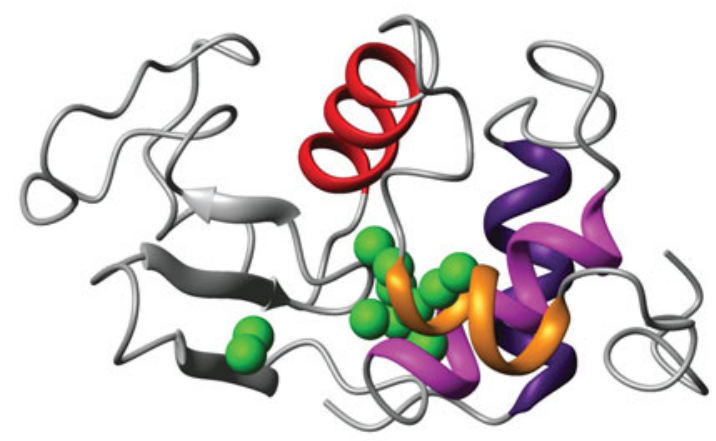

Fig. 3. Interaction between human lysozyme and disaccharide 1, observed by NMR. (a) Selected regions from the 2D NOESY spectra of pure human lysozyme (red) and a 1:1 mixture with disaccharide 1 (black) demonstrate some of the shift changes observed after the addition of disaccharide 1. Peak labeling is as described in Fig. 2b. (b) Mapping of resonances with chemical shift changes exceeding 0.07 ppm (online Table S1) after the addition of disaccharide $\mathbf{1}$ onto the 3D structure of lysozyme; the atoms are indicated as green spheres. The structure has an identical orientation and helix coloring to that described in Fig. $1 a$.

d. The next largest shift changes concerned the Trp109 side chain, which is an important component of the binding site surface and exhibited direct interactions with Glu35; the shortest distance between these side chains is $2 \cdot 2 \AA$.

In addition to the well-known relative motions of the two domains required for ligand binding, HL undergoes a series of specific processes, both chemical and structural, when the $\mathrm{pH}$ is varied near 6.8. These processes include large parts of the $\alpha$-domain with the catalytic Glu35, the side chain of Trp109, most of the b and d helices, and the loop between the second and third $\beta$-strands from the $\beta$-domain (Fig. 2 c). The identical pKa (within the measurement error) of all relevant resonances strongly indicated that all of these events are coupled (online Table S1, Supplementary Information). Although some strong interactions with Glu35 have been previously reported (Kuramitsu et al. 1974) (e.g. with Trp109) or can be assumed on the basis of their proximities (e.g. to the amide of Ala 111), other titrating residues appeared to be too far away from Glu35 to show direct effects due to the charge change upon (de)protonation; reports have instead focused on a higher flexibility of the $\alpha$-domain that involves mutual rearrangements between helices $b$ and $d$, which mirrors similar observations regarding factors such as ligand binding, temperature or pressure variations (Refaee et al. 2003; Young et al. 1994). The relative position of helices b and $\mathrm{d}$ within the $\alpha$-domain modulated by the hydrogen bonding network with Glu35, Trp109 and Ala111 is further defined by the previously described interaction between Arg115 and Trp34, and the Arg115Glu mutation has been reported to modify both the position of helix $\mathrm{d}$ and the enzyme activity (Harata et al. 1998). Thus, (de)protonation of Glu35 appears to trigger processes that spread over most of the $\alpha$-domain.

\subsection{Interaction between HL and disaccharide 1, observed by NMR}

The binding sites of the c-type lysozyme include six individual subsites (labeled A-F, Fig. 1a) for specific interactions with multiple saccharide rings (Chipman \& Sharon, 1969). Mixtures of HL and disaccharide 1 (Fig. 1d) yield observable chemical shift value changes at only $\mathrm{pH} 5 \cdot 5$; effects caused by intermolecular interactions were undetectable at $\mathrm{pH}$ 3.8. At $\mathrm{pH} 5 \cdot 5$, the nuclei in the residues surrounding the D-F sub-sites that show changes were Glu35 $\mathrm{HN}$ and $\mathrm{H} \gamma$, Asn $44 \mathrm{H}$, Trp109 $\mathrm{H} \delta 1$ and $\mathrm{H} \epsilon 1$, and Arg113 HN (Fig. 3a).

The affected residues were mapped onto the $3 \mathrm{D}$ structure of HL in Fig. $3 b$ and demonstrated transient binding at subsites $\mathrm{D}$ and $\mathrm{E}$ (Fig. 1a). Helices $\mathrm{b}$ and $\mathrm{d}$ and the catalytic Glu35 in particular responded to the addition of disaccharide 1 . Furthermore, a chemical shift change was observed on the $\beta$-domain in the center of the first strand (Asn44). Since Trp109 is part of the binding cleft (Fig. 1a), it is unsurprising that the aforementioned structural changes mediated by this tryptophan were affected by ligand binding. The observations of chemical shift changes for Arg113, whose location was distal to all binding sites and buried behind the preceding helix loop (residues 110-111), were in agreement with a coupling between the ligand-binding and conformational effects.

\subsection{Molecular modeling of the interaction between $H L$ and disaccharide 1}

Molecular modeling (Kar et al. 2016; Zhang et al. 2016) and data mining (Lütteke et al. 2005; Rojas-Macias \& Lütteke, 2015) tools are necessary for general discussions of the principles of carbohydrate-protein interactions. In order to gain insight into the binding mode for disaccharide $\mathbf{1}$, molecular 
modeling is a rational tool to use. During the docking, each simulation includes 100 runs, which generate 100 conformers for the ligand. The top 10 conformers with low energy were selected for further analysis. For this step, both energy and ligand binding were considered. In fact, in the end, only the conformer with the lowest energy was selected for interaction description, which also shows a rational binding conformation. Figure $4 a$ displays the most favorable energetic structure of HL and disaccharide 1. A close-up view of the basic pocket with the LPS-interacting side chains is presented in Fig. 4b. Red dashed lines indicate the hydrogen bonds. The simulation of the binding for disaccharide $\mathbf{1}$ indicated its interaction with $\mathrm{HL}$ at the substrate binding sites $\mathrm{C}$ and $\mathrm{D}$. This observation is in agreement with the analysis based on the crystal structure of HL with the tetrasaccharide 2 since the binding sites $\mathrm{C}$ and especially D provided most of the interactions between sugar and protein. Comparisons of this specific result with similar cases in the PDB uncovered numerous meaningful agreements. The protein-carbohydrate interaction analysis in the PDB revealed that Trp, Tyr, Asp, Asn and His were the most overrepresented amino acids in the vicinity of $\alpha$-D-Galp residues (Fig. 4c). The previously described data mining approach (Bhunia et al. 2010; Lütteke et al. 2005) provided important information regarding the amino acid residues that typically occur in the vicinity of $\alpha$-D-Galp. Using our data mining protocols, we performed an overview of the molecular interactions between $\alpha$-D-Gal $p$ and the functional groups of certain amino acid residues (Fig. 4c) in relation to the general structural aspects of lysozyme-carbohydrate interactions.

Molecular modeling calculations with respect to the electrostatic surface potentials and hydrophobic patches were also performed (online Fig. S4). The differences in the patterns of the electrostatic surface potentials suggest variations in ligand binding and deviations in the aggregation/fibrillation behavior between human and avian lysozyme.

\subsection{X-ray crystallography-based study of the interaction between HL and tetrasaccharide 2}

To provide data that are independent of the results of the NMR measurements and molecular modeling calculations, X-ray crystallographic experiments were performed for human lysozyme. Extensive experiments to co-crystallize $\mathrm{HL}$ with bound disaccharide $\mathbf{1}$ (Fig. 1d) at different $\mathrm{pH}$ values failed. We did not observe a convincing electron density for bound disaccharide, even after the crystals were soaked with high disaccharide concentrations. We assume that the disaccharide was too short to provide sufficient interaction opportunities with the protein to form a stable complex. Therefore, the study of a longer oligosaccharide ligand representing a larger polysaccharide fragment was initiated.
The crystal complex of HL with tetrasaccharide 2 was successfully obtained by co-crystallization of $1.9 \mathrm{mM} \mathrm{HL}$ in the presence of $20 \mathrm{mM}$ tetrasaccharide 2 . The X-ray diffraction resolution of the crystals was approximately $1.0 \AA$. A summary of the data collection and structure refinement is presented in Table 1. For details regarding the definitions of the individual parameters, see online Table S2 in the Supplementary Information.

The refined structure of HL in complex with tetrasaccharide 2 reveals the binding of tetrasaccharide 2 near the A, B, C, and $\mathrm{D}$ substrate-binding sites of the enzyme (Fig. 5). The Galf-I furanoside unit of tetrasaccharide $\mathbf{2}$ is located near site A, and the Galp-II pyranoside unit is located near site $\mathrm{B}$. The second repeating unit (Galf-III)-(Galp-IV) is in proximity to sites C and D. Both Galp units of tetrasaccharide 2 adopt a chair conformation. Overall, there are eight direct hydrogen bonds between tetrasaccharide 2 and the amino acid residues of HL. Seven residues (Ile59, Asn60, Tyr63, Trp64, Ala76, Asp102, and Trp109) form hydrophobic interactions. Through comparison with tetra $\mathrm{N}$-acetyl$\mathrm{D}$-glucosamine, it is clear that the sugar ring systems of tetrasaccharide $\mathbf{2}$ are not identically positioned. However, a similar number of direct hydrogen bonds, hydrophobic interactions and water-mediated bridged hydrogen bonds jointly contribute to the overall binding affinity.

Based on number and ratio, an important contribution to the overall binding affinity for tetrasaccharide 2 seems to be provided by bridged hydrogen bonds that are mediated by approximately 20 water molecules within the binding pocket toward the following lysozyme residues: Glu35, Asp49, Asp53, Asn60, Tyr63, Val99, Arg98, Gly105, Ala108, Val110, and Ala111 (Fig. 6 and online Table S3, Supplementary Information). This ratio and mix of interactions is similar to those observed for (GlcNAc) $)_{4}$ (PDB entry 1LZR) and (GlcNAc) $)_{4} /(\mathrm{GlcNAc})_{2}$ (PDB entry 1LZS) (Song et al. 1994) bound to HL. The superposition of HL-2 with $\mathrm{HL}-(\mathrm{GlcNAc})_{4}$ and with HL-(GlcNAc) $)_{4} /(\mathrm{GlcNAc})_{2}$ resulted in RMSD values of 0.23 and $0.46 \AA$, respectively (based on $130 \mathrm{C}$-alpha positions), thus indicating that the $3 \mathrm{D}$ structures of the protein-ligand complexes are similar in all three complexes.

The binding modes of tetrasaccharides 2 and (GlcNAc) $)_{4}$ in the A, B, C, and D sites display small but significant differences. Specifically, Arg98 forms a direct hydrogen bond between its side-chain atom, $\mathrm{N} \varepsilon 2$, and the $\mathrm{O} 1$ atom of the Galf-I unit in site A of the HL-2 complex. Additionally, water molecules mediate a bridged hydrogen bond between the $\mathrm{NH}$ group of Arg98 and the OH group of Galf-I. Arg98 is not involved in the binding of $(\mathrm{GlcNAc})_{4}$ or $(\mathrm{GlcNAc})_{2}$ to HL.

The major interactions of the Galp-II unit in site B of HL are managed by direct hydrogen bonds with the surrounding amino acid residues Tyr63, Asp102, and Gln104. In addition to the hydrogen bond between atom $\mathrm{O} 7$ and the $\mathrm{OH}$ group 
(a)

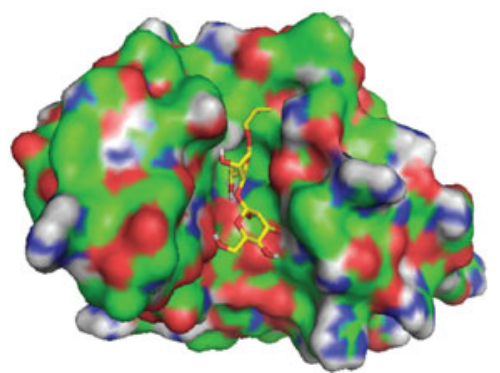

(b)

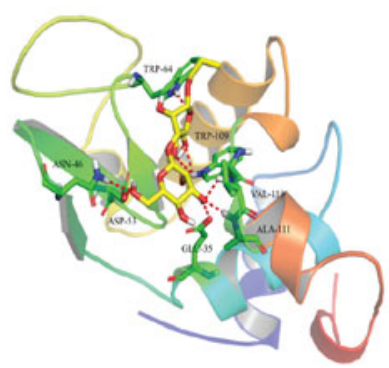

$(c)$

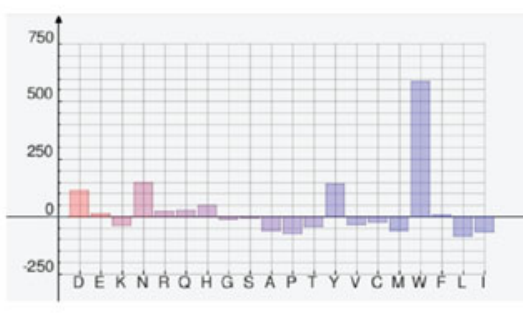

Fig. 4. Molecular modeling of the interaction between human lysozyme and disaccharide 1. (a) Molecular surface of lysozyme with carbons (green), oxygens (red), nitrogens (blue) and polar hydrogens (gray). (b) Close-up view of the basic pocket with disaccharide 1 shown in stick rendering and with hydrogen bonds indicated by red dashed lines. (c) Amino acid residues in the vicinity of $\alpha$-Gal in the protein-carbohydrate complexes deposited in the PDB, which indicates the deviation from natural abundance. Trp, Tyr, Asp, and His are overrepresented by greater than $100 \%$ (i.e. they are observed twice as often or more in a $4 \AA$ radius of $\alpha$-Gal compared with an average protein).

Table 1. Data collection and refinement statistics

\begin{tabular}{ll}
\hline PDB entry & 5 LSH \\
\hline Data collection & \\
Temperature $(\mathrm{K})$ & 100 \\
Resolution range $(\AA)^{\mathrm{a}}$ & $29 \cdot 05-1 \cdot 061(1 \cdot 099-1 \cdot 061)$ \\
Space group & $P 2(1) 2(1) 2(1)$ \\
Unit cell a, b, c $(\AA)$ & $33 \cdot 1,56 \cdot 0,60 \cdot 5$ \\
Multiplicity & $11 \cdot 9(9 \cdot 8)$ \\
Completeness $(\%)^{\mathrm{a}}$ & $92(69)$ \\
Mean $(I) / \sigma(I)^{\mathrm{a}}$ & $17 \cdot 06(1 \cdot 52)$ \\
$R_{\text {p.i.m. }}(\%)^{\mathrm{a}, \mathrm{b}}$ & $7 \cdot 6(108 \cdot 8)$ \\
CC $(1 / 2)(\%)^{\mathrm{a}, \mathrm{b}}$ & $100(63 \cdot 3)$ \\
Refinement & \\
R-work $(\%)^{\mathrm{a}, \mathrm{b}}$ & $18 \cdot 1(32 \cdot 4)$ \\
R-free $(\%)^{\mathrm{a}, \mathrm{b}}$ & $20 \cdot 4(33 \cdot 6)$ \\
$B$-factors $\left(\AA^{2}\right)($ No. of non-hydrogen & \\
atoms) & \\
All & $11 \cdot 4(1414)$ \\
Ligand KTS & $16 \cdot 6(90)$ \\
Water molecules & $20 \cdot 9(160)$ \\
rmsd (bonds) $(\AA)$ & $0 \cdot 013$ \\
rmsd (angle) $\left({ }^{\circ}\right)$ & $1 \cdot 58$ \\
Rotamer outliers (\%) & $1 \cdot 7$ \\
Ramachandran plot statistics (\%) & \\
Favored & $97 \cdot 0$ \\
Allowed & $2 \cdot 8$ \\
Outliers & $0 \cdot 0$ \\
&
\end{tabular}

${ }^{\mathrm{a}}$ Values in parentheses are for the high-resolution shell.

${ }^{\mathrm{b}}$ For details regarding the definitions of the individual parameters, see online Table S2 in the Supplementary Information.

of Tyr63, the aromatic plane of Tyr63 provides a strong hydrophobic interaction with the Galp-II moiety. A similar interaction of Tyr63 with the second GlcNAc residue is present within the HL-(GlcNAc) ${ }_{4}$ complex. Notably, the orientation of the Tyr63 side chain in the HL-2 complex is rotated by ca. $10^{\circ}$ for an optimal interaction with the shifted sugar position in comparison with the GlcNAc unit. The $\mathrm{O} \delta 2$ side-chain atom of the Asp102 residue contributes two hydrogen bonds to the binding of the first two sugar units of 2, but only one direct hydrogen bond to the first GlcNAc unit is observed in $(\mathrm{GlcNAc})_{4}$. In the HL$(\mathrm{GlcNAc})_{4} /(\mathrm{GlcNAc})_{2}$ complex, atom $\mathrm{O} \delta 1$ forms two hydrogen bonds with atoms $\mathrm{N}$ and $\mathrm{O} 6$ of the first GlcNAc moiety of $(\mathrm{GlcNAc})_{4} /(\mathrm{GlcNAc})_{2}$. As a consequence of these differences, tetrasaccharide 2 adopts a closer binding orientation toward the 'bottom' of the binding pocket, as compared with $(\mathrm{GlcNAc})_{4}$ and $(\mathrm{GlcNAc})_{4} /(\mathrm{GlcNAc})_{2}$.

The Galf-III unit is located near the $\mathrm{C}$ substrate-binding site. Only one direct hydrogen bond is formed between the Trp64 atom Ne1 and atom O19. Similar hydrogen bonds are contributed by these two residues for sugar binding in the HL complex to produce $\mathrm{HL}-(\mathrm{GlcNAc})_{4}$ and HL$(\mathrm{GlcNAc})_{4} /(\mathrm{GlcNAc})_{2}$. The unit Galp-IV is located between the two substrate-binding sites, $\mathrm{C}$ and $\mathrm{D}$. The only two direct hydrogen bonds are formed between atom $\mathrm{O} 16$ and the main-chain atom $\mathrm{O}$ of $\mathrm{Gln} 58$ and between atom $\mathrm{O} 15$ and the main-chain atom $\mathrm{N}$ of Asn60. Within the HL(GlcNAc) ${ }_{4}$ complex, a similar hydrogen bond is contributed by Gln58 to the fourth GlcNAc moiety. Additionally, two residues, Asn46 and Asp53, form direct hydrogen bonds with GlcNAc atom $\mathrm{O} 1$ in the $\mathrm{HL}-(\mathrm{GlcNAc})_{4}$ complex. Interestingly, in the $\mathrm{HL}-(\mathrm{GlcNAc})_{4} /(\mathrm{GlcNAc})_{2}$ complex, Gln58 is not involved in the interaction with the fourth GlcNAc moiety; instead, it is involved in the interaction with the Asn46 side chain and the main-chain atoms of Ala108 and Val110. Notably, approximately 10 water molecules play an important role in mediating interactions between unit Galp-IV of tetrasaccharide 2 and the Glu35, Asp49, Ser51, Asp53, Gln58, Gln104, Ala108, and Ala111 residues near the $\mathrm{D}$ and $\mathrm{E}$ substrate-binding sites. It is possible that a fifth sugar moiety may be adopted within this interaction network, which would contribute to a further gain in binding affinity. 
(a)

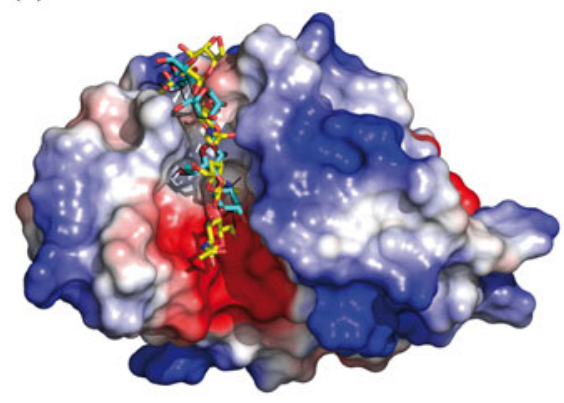

(b)

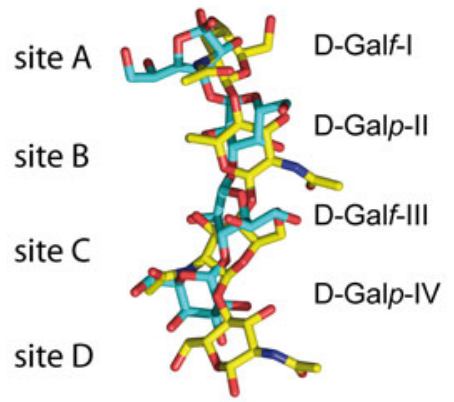

(c)

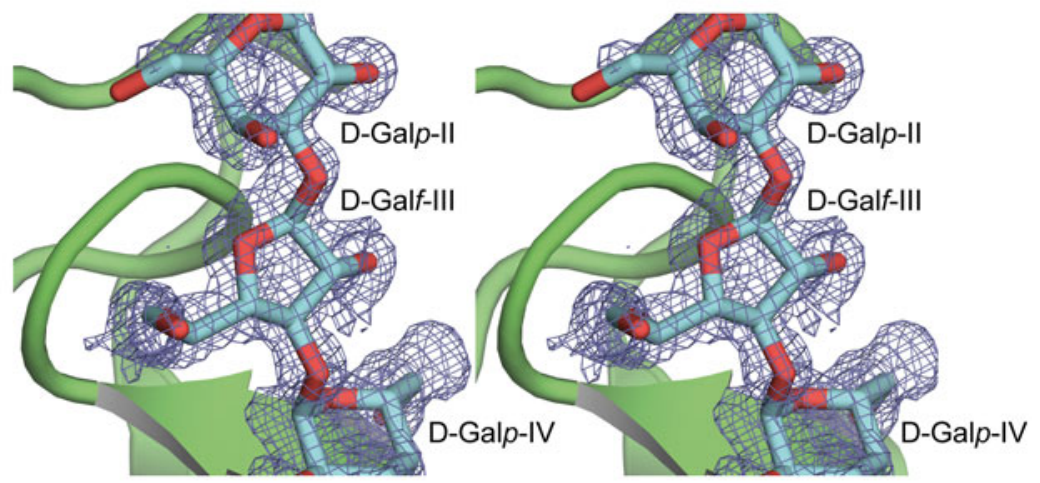

Fig. 5. Molecular surface of HL in the complex with tetrasaccharide 2. (a) Representation of the molecular surface of HL is colored according to the electrostatic potential. Tetrasaccharide $\mathbf{2}$ is represented by sticks for one conformation (carbon atoms in cyan and oxygen atoms in red) and by black lines for an alternative conformation. The HL (PDB-entry 1LZR)-bound chitotetraose (GlcNAc) 4 ligand is superimposed and represented by sticks (carbon atoms in yellow). (b) Close-up view of the superimposed ligands, tetrasaccharide 2 (carbon atoms in cyan) and (GlcNAc) 4 (carbon atoms in yellow, oxygen atoms in red and nitrogen atoms in blue. (c) Representation in stereo of the electron density $2 \mathrm{~F}_{\mathrm{obs}}-1 \mathrm{~F}_{\text {calc }}$ omit map defining bound tetrasaccharide 2 . The Galp-II, Galf-III and Galp-IV units are represented by sticks (carbon atoms in cyan and oxygen atoms in red). The protein backbone is indicated by a ribbon representation in green. The representation was generated using PyMOL v.1.6 (DeLano, 2002).

\section{Discussion}

This study is the first to indicate that tetrasaccharide $\mathbf{2}$, which represents part of the $\mathrm{O}$-chain of the K. pneumoniae O1 LPS, binds to HL at its conserved sites (A, B, C, and D) within the substrate binding pocket; this binding occurs mainly through direct hydrogen bonds and indirect hydrogen bonds that are mediated by water molecules. Our SPR experiments indicated a specific binding of LPS fragments to lysozyme, however with a rather low on-rate. A couple of synchronously occurring processes have to be considered for the evaluation of this slow on-rate: closing/opening of the binding cleft, rearrangement of side chains, water molecules have to be squeezed out of the binding cleft before the sugar molecule can bind and finally the sugar has to adopt an optimal conformation. In the case of dihydrofolate reductase the dynamic events during catalysis were deciphered e.g. by NMR experiments and helped to model the energy landscape (Boehr et al. 2006). We have performed a combination of molecular dynamic simulation, NMRspectroscopy, and X-ray crystallography to thoroughly characterize the ligand binding. Our NMR titrations provide a detailed picture of how the active site and the overall structure are related to each other. At $\mathrm{pH} 6 \cdot 8$, deprotonation of Glu35 occurs, which inactivates the enzyme at this unusually high pKa value, and helices b (with Glu35) and d undergo a substantial repositioning as indicated by the mapping of $\mathrm{pH}$ titration effects in Fig. $2 c$. Because one of the first catalysis steps also involves the deprotonation of Glu35, these domain-wide structural changes are likely to modulate the continuation of the catalysis reaction. Together with earlier studies (Refaee et al. 2003; Young et al. 1994), our data indicate a general flexibility and lower stability of helix d, which can be affected by numerous factors, including sugar binding and temperature changes. The ability of lysozyme to exhibit complex responses to environmental changes is evidenced by the following observations: the titration effects observed for the proton probes on Glu35, Trp109 and Ala111; the line broadening of local motions that involve Trp109 and Ala111; the spatial neighborhood of these three residues, which allows for direct interactions; and the wide range of titrating resonances on helices $b$ and $d$. These structural rearrangements appear to be strongly coupled to enzyme activation by the (de)protonation of Glu35.

The carbohydrate binding site of HL displays a strong structural plasticity. This fact agrees with earlier observations by NMR relaxation studies, that the binding of carbohydrates 


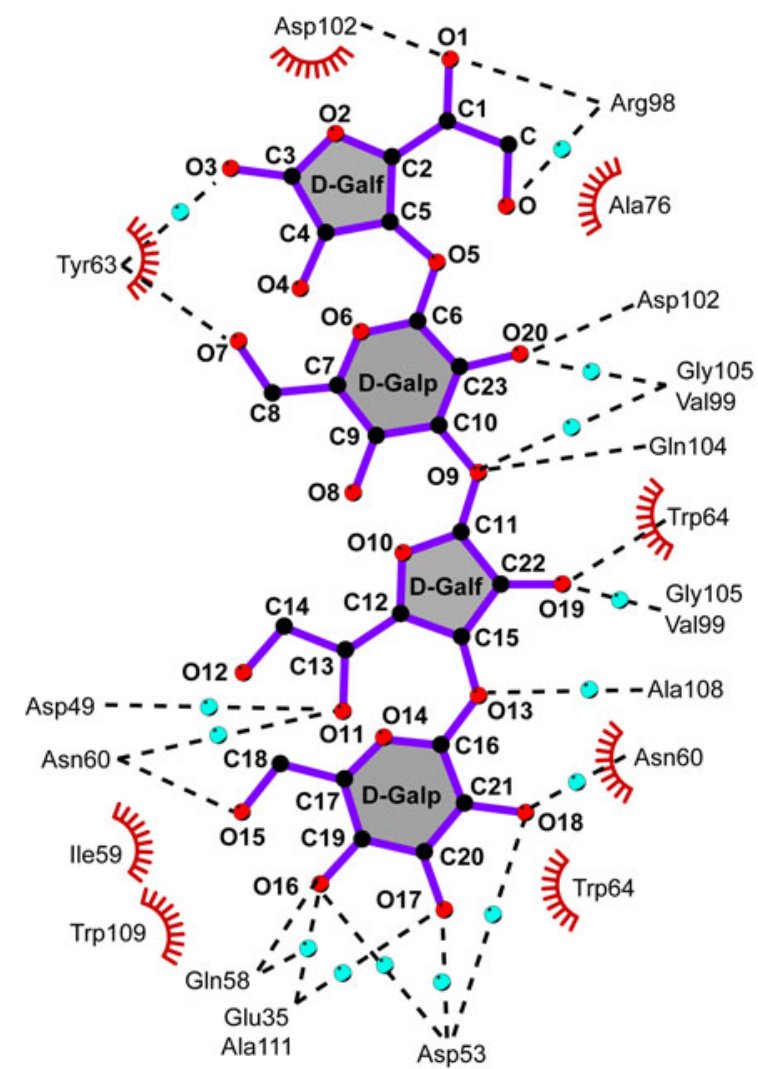

Fig. 6. Binding site of human lysozyme with bound tetrasaccharide 2. The ligand is shown as a ball-and-stick representation; the bonds are indicated in purple. The protein residues are represented without side chains. Hydrogen bonds are shown as black dashed lines, and the spoked arcs represent protein residues that form hydrophobic interactions with the ligand. The cyan spheres indicate water molecules, which provide bridged hydrogen bonds between the ligand atoms and amino acid residues. The individual interactions are provided in online Table S3 of the Supplementary Information. The representation was derived from an analysis with LigPlot+ (Laskowski \& Swindells, 2011).

to proteins is significantly determined by changes in conformational entropy (see for example Diehl et al. 2010). This may take various forms, for example of transient interactions such as water-bridged hydrogen bonds, or of changes in protein dynamics without observation of structural changes in crystal structures. Only a few amino acid residues are in direct hydrogen-bonding contact with the oligosaccharide chain. Most interactions are formed by hydrophobic contacts and particularly by water-mediated bridged hydrogen bonds. These latter two modes of interaction are less constraining and can be used to adopt binding environments for various ligands. The lectin-like ability of HL to interact with the O-chain of bacterial LPS highlights the strong possibility of a new role of HL in immune defense functions. This study may enable future developments of new and important therapeutic approaches to prevent and treat bacterial infections.

\subsection{Speculation}

The investigated lectin-like ability of HL to interact with the O-polysaccharide chain of bacterial LPS shows the possibility of an unknown glycan-guided mechanism of lysozyme's biological role. It underlies recognition of the bacterial cell wall by lysozyme and may complement its known immune defense functions. Further investigation of carbohydrate specificity of lysozyme with the use of larger linear and branched oligosaccharide ligands related to the O-chain of K. pneumoniae $\mathrm{O} 1$ as well as of antigenic polysaccharides of others bacterial pathogens may show the scope and limitations of the studied phenomenon.

\section{Supplementary material}

The supplementary material for this article can be found at https://doi.org/10.1017/S0033583517000075.

\section{Acknowledgements}

The Swedish NMR Centre is acknowledged for supplying instrument time and support. Diffraction data were collected on a P14 operated by EMBL at the PETRAIII storage ring (Hamburg, Germany). We are grateful to the beamline staff for providing assistance in using the beamline. We thank Dr Timothy Weaver (Cincinnati Children's Hospital Medical Center, Cincinnati, USA) for the provision of human recombinant lysozyme.

\section{Financial support}

This work was supported by the King Abdullah University of Science and Technology (grant KUK-11-008-23 awarded to B.N. with a Ph.D. position for L.W.) and the European Research Council (ERC-2008-AdG 227700 to B.N.). Beamtime on the P14 at the EMBL outstation in Hamburg was funded by a BioStruct-X grant. We thank the Sialic Acids Society for financial support. The synthetic portion of the work was supported by the RSF (grant 14-23-00199 to N.E.N.). A.D. would like to thank CSIR, Govt. of India for senior research fellowship.

\section{Conflict of interest}

None

\section{References}

Adams, P. D., Afonine, P. V., Bunkóczi, G., Chen, V. B., Davis, I. W., Echols, N., Headd, J. J., Hung, L.-W., Kapral, G. J., GrosseKunstleve, R. W., McCoy, A. J., Moriarty, N. W., Oeffner, R., Read, R. J., Richardson, D. C., Richardson, J. S., Terwilliger, T. C. \& ZWART, P.H. (2010). PHENIX: a comprehensive Pythonbased system for macromolecular structure solution. Acta 
Crystallographica Section D: Biological Crystallography 66(2), 213-221.

Akinbi, H. T., Epaud, R., Bhatt, H. \& Weaver, T. E. (2000). Bacterial killing is enhanced by expression of lysozyme in the lungs of transgenic mice. Journal of Immunology 165, 5760-5766.

Bartels, C., Xia, T. H., Billeter, M., Gưntert, P. \& Wüthrich, K. (1995). The program XEASY for computer-supported NMR spectral analysis of biological macromolecules. Journal of Biomolecular NMR 6, 1-10.

Bhunia, A., Vivekanandan, S., Eckert, T., Burg-Roderfeld, M., Wechselberger, R., Romanuka, J., Bächle, D., Kornilov, A. V., von der Lieth, C.-W., Jiménez-Barbero, J., Nifantiev, N. E., Schachner, M., Sewald, N., Lưtteke, T., Gabius, H.-J. \& Siebert, H.-Ch. (2010). Why structurally different cyclic peptides can be glycomimetics of the HNK-1 carbohydrate antigen. Journal of the American Chemical Society 132, 96-105.

Boenr, D. D., McElheny, D., Dyson, H. J. \& Wright, P.E. (2006) The dynamic energy landscape of dihydrofolate reductase catalysis. Science 313, 1638-1642.

Chipman, D. M. \& Sharon, N. (1969). Mechanism of lysozyme action. Science 165, 454-465.

Claridge, T. (2009). Software review of MNova: NMR data processing, analysis, and prediction software. Journal of Chemical Information and Modeling 49, 1136-1137.

Delaglio, F., Grzesiek, S., Vuister, G. W., Zhu, G., Pfeifer, J. \& Bax, A. D. (1995). NMRPipe: a multidimensional spectral processing system based on UNIX pipes. Journal of Biomolecular NMR 6, 277-293.

DeLano, W. L. (2002). The PyMOL molecular graphics system. (DeLano Scientific, San Carlos, California, USA, 2002). http:// www.pymol.org.

Diehl, C., Engström, O., Delaine, T., Håkansson, M., Genheden, S., Modig, K., Leffler, H., Ryde, U., Nilsson, U. J. \& AKKe, M. (2010). Protein flexibility and conformational entropy in ligand design targeting the carbohydrate recognition domain of galectin-3. Journal of the American Chemical Society 132, 14577-14589.

Emsley, P., LohKamp, B., Scott, W. G. \& Cowtan, K. (2010). Features and development of Coot. Acta Crystallographica Section D: Biological Crystallography 66, 486-501.

Enani, M. A. (2015). Antimicrobial resistance. Insights from the declaration of world alliance against antibiotic resistance. Saudi medical Journal 36, 11-12.

Enani, M. A. \& El-Khizzi, N. A. (2012). Community acquired Klebsiella pneumoniae, K1 serotype. Invasive liver abscess with bacteremia and endophthalmitis. Saudi medical Journal 33, 782-786.

GuptA, A. (2002). Hospital-acquired infections in the neonatal intensive care unit-Klebsiella pneumoniae. Seminars in Perinatology 26, 340-345.

Harata, K., Abe, Y. \& Muraki, M. (1998). Full-matrix least-squares refinement of lysozymes and analysis of anisotropic thermal motion. Proteins: Structure, Function, and Bioinformatics 30, 232-243.

$\mathrm{K}_{\mathrm{ABSCH}}, \mathrm{W}$. (2010). Integration, scaling, space-group assignment and post-refinement. Acta Crystallographica Section D: Biological Crystallography 66(2), 133-144.

Kar, R. K., Gazova, Z., Bednarikova, Z., Mrove, K. H., Ghosh, A., Zhang, R., Ulicna, K., Sieber, H.-Ch., Nifantiev, N. E. \& BHUNiA, A. (2016). Evidence for Inhibition of lysozyme amyloid fibrillization by peptide fragments from human lysozyme: a combined spectroscopy, microscopy, and docking study. Biomacromolecules 17, 1998-2009.

Koradi, R., Billeter, M. \& WÜthrich, K. (1996). MOLMOL: a program for display and analysis of macromolecular structures. Journal of Molecular Graphics 14, 51-55.

Krylov, V.B., Argunov, D. A., Vinnitskiy, D.Z., Gerbst, A. G., Ustyuzhanina, N.E., DMitrenoK, A.S. \& Nifantiev, N.E. (2016). The pyranoside-into-furanoside rearrangement of alkyl glycosides: scope and limitations. Synlett 27, 1659-1664.

Krylov, V. B., Argunov, D. A., Vinnitskiy, D. Z., Verkhnyatskaya, S. A., Gerbst, A. G., Ustyuzhanina, N. E., Dmitrenok, A. S., Huebner, J., Holst, O., Siebert, H.-Ch \& Nifantiev, N. E. (2014). Pyranoside-into-furanoside rearrangement: new reaction in carbohydrate chemistry and its application in oligosaccharide synthesis. Chemistry-A European Journal 20, 16516-16522.

Kuramitsu, S., Ikeda, K., Hamaguchi, K., Fujio, H., Amano, T., Shiro, M. I. W. A. \& NishinA, T. (1974). Ionization constants of Glu 35 and Asp 52 in hen, turkey, and human lysozymes. Journal of Biochemistry 76, 671-683.

Laskowski, R. A. \& Swindells, M.B. (2011). LigPlott: multiple ligand-protein interaction diagrams for drug discovery. Journal of Chemical Information and Modeling 51, 2778-2786.

Lee-Huang, S., Maiorov, V., Huang, P. L., Ng, A., Lee, H. C., Chang, Y. T., Kallenbach, N., HuAng, P. L. \& Chen, H. C. (2005). Structural and functional modeling of human lysozyme reveals a unique nonapeptide, HL9, with anti-HIV activity. Biochemistry 44, 4648-4655.

LÜtTeKE, T., Frank, M. \& VON DER Lieth, C. W. (2005). Carbohydrate structure suite (CSS): analysis of carbohydrate 3D structures derived from the PDB. Nucleic Acids Research 33, D242-D246.

Markart, P., Korfhagen, T. R., Weaver, T. E. \& Akinbi, H. T. (2004). Mouse lysozyme $\mathrm{M}$ is important in pulmonary host defense against Klebsiella pneumoniae infection. American Journal of Respiratory and Critical Care Medicine 169, 454-458.

MasschalCK, B. \& Michiels, C. W. (2003). Antimicrobial properties of lysozyme in relation to foodborne vegetative bacteria. Critical Reviews in Microbiology 29, 191-214.

Muraki, M., Harata, K., Sugita, N. \& Sato, K. I. (1996). Origin of carbohydrate recognition specificity of human lysozyme revealed by affinity labeling. Biochemistry 35, 13562-13567.

Murshudov, G. N., Skubák, P., Lebedev, A. A., Pannu, N. S., Steiner, R. A., Nicholls, R. A., Winn, M.D., Long, F. \& Vagin, A. A. (2011). REFMAC5 for the refinement of macromolecular crystal structures. Acta Crystallographica Section D: Biological Crystallography 67, 355-367.

OHNo, N. \& MORRISON, D. C. (1989a). Lipopolysaccharide interactions with lysozyme differentially affect lipopolysaccharide immunostimulatory activity. European Journal of Biochemistry 186, 629-636.

Ohno, N. \& Morrison, D. C. (1989b). Lipopolysaccharide interaction with lysozyme. Binding of lipopolysaccharide to lysozyme and inhibition of lysozyme enzymatic activity. Journal of Biological Chemistry 264, 4434-4441.

Osserman, E. F., Klockars, M. A. T. T. I., Halper, J. A. M. E. S. \& FisCHel, R. E. (1973). Effects of lysozyme on normal and transformed mammalian cells. Nature 243, 331-335.

Refaee, M., Tezuka, T., Akasaka, K. \& Williamson, M.P. (2003). Pressure-dependent changes in the solution structure of hen egg white lysozyme. Journal of Molecular Biology 327, 857-865. 
Rojas-Macias, M. A. \& LÜTTEKe, T. (2015). Statistical analysis of amino acids in the vicinity of carbohydrate residues performed by GlyVicinity. Methods in Molecular Biology 1273, 215-226.

Shin, H. J., Lee, H., Park, J. D., Hyun, H. C., Sohn, H. O., Lee, D. W. \& KIM, Y.S. (2007). Kinetics of binding of LPS to recombinant CD14, TLR4, and MD-2 proteins. Molecules and Cells 24, $119-124$.

Simicki, A. K., Klein, P., Morand, M., Kiczka, W. \& Studnicka, M. (1998). Immunostimulatory effects of dimerized lysozyme (KLP-602) on the nonspecific defense mechanisms and protection against furunculosis in salmonids. Veterinary Immunology and Immunopathology 61, 369-378.

Song, H., Inaka, K., Maenaka, K. \& Matsushima, M. (1994). Structural changes of active site cleft and different saccharide binding modes in human lysozyme co-crystallized with hexa-N-acetyl-chitohexaose at $\mathrm{pH}$ 4.0. Journal of Molecular Biology 244, 522-540.

Travis, S. M., Conway, B. A. D., Zabner, J., Smith, J. J., Anderson, N. N., Singh, P. K., Greenberg, E. P. \& Welsh, M. J. (1999). Activity of abundant antimicrobials of the human airway. American Journal of Respiratory Cell and Molecular Biology 20, 872-879.

Tsvetrov, Y. E., Burg-Roderfeld, M., Loers, G., ArdÁ, A., Sukhova, E. V., Khatuntseva, E. A., Grachev, A. A., Chizhov, A. O., Siebert, H.-Ch., Schachner, M., Jiménez-Barbero, J. \& Nifantiev, N.E. (2012). Synthesis and molecular recognition studies of the HNK-1 trisaccharide and related oligosaccharides. The specificity of monoclonal anti-HNK-1 antibodies as assessed by surface plasmon resonance and STD NMR. Journal of the American Chemical Society 134, 426-435.

Ulrich, E. L., Akutsu, H., Doreleijers, J. F., Harano, Y., Ioannidis, Y. E., Lin, J., Livny, M., Mading, S., Maziuk, D., Miller, Z., Nakatani, E., Schulte, C. F., Tolmie, D. E., Wenger, R. K., Yao, H. \& Markley, J.L. (2008). BioMagResBank. Nucleic Acids Research 36, D402-D408.
Vagin, A. \& Teplyakov, A. (1997). MOLREP: an automated program for molecular replacement. Journal of Applied Crystallography 30, 1022-1025.

Verkhnyatskaya, S. A., Krylov, V.B. \& Nifantiev, N. E. (2017). Pyranoside-into-furanoside rearrangement of 4-pentenyl glycoside in the synthesis of tetrasaccharide related to galactan I of Klebsiella pneumoniae. European Journal of Organic Chemistry 2017, 710-718.

Vranken, W. F., Boucher, W., Stevens, T. J., Fogh, R. H., Pajon, A., Llinas, M., Ulrich, E. L., Markley, J. L., Ionides, J. \& LaUe, E. D. (2005). The CCPN data model for NMR spectroscopy: development of a software pipeline. Proteins: Structure, Function, and Bioinformatics 59, 687-696.

Winn, M. D., Ballard, C. C., Cowtan, K. D., Dodson, E. J., Emsley, P., Evans, P. R., Keegan, R. M., Krissinel, E. B., Leslie, A. G. W. McCoy, A., McNicholas, S. J., Murshudov, G. N., Pannu, N. S., Potterton, E.A., Powell, H. R., Read, R. J., Vagin, A. \& WiLSON, K. S. (2011). Overview of the CCP4 suite and current developments. Acta Crystallographica Section D: Biological Crystallography, 67, 235-242.

Young, A. C., Tilton, R. F. \& Dewan, J. C. (1994). Thermal expansion of hen egg-white lysozyme: comparison of the $1.9 \AA$ resolution structures of the tetragonal form of the enzyme at $100 \mathrm{~K}$ and 298 K. Journal of Molecular Biology, 235, 302-317.

Young, N. M., Gidney, M. A. J., Gudmundsson, B. E., MacKenzie, C. R., To, R., Watson, D. C. \& BundLE, D. R. (1999). Molecular basis for the lack of mimicry of Brucella polysaccharide antigens by $\mathrm{Ab} 2 \gamma$ antibodies. Molecular Immunology 36, 339-347.

Zhang, R., Eckert, T., Lutteke, T., Hanstein, S., Scheidig, A. J., Bonvin, A., Nifantiev, N. E., Kozar, T., Schauer, R., Enani, M. A. \& Siebert, H.C. (2016). Structure-function relationships of antimicrobial peptides and proteins with respect to contact molecules on pathogen surfaces. Current Topics in Medicinal Chemistry 16, 89-98. 